\title{
OPEN Spin glass behavior and magnetic boson peak in a structural glass of a magnetic ionic liquid
}

\begin{abstract}
Maiko Kofu ${ }^{1 凶}$, Ryuta Watanuki ${ }^{\bowtie}$, Toshiro Sakakibara ${ }^{3}$, Seiko Ohira-Kawamura $^{1}$, Kenji Nakajima ${ }^{1}$, Masato Matsuura ${ }^{4}$, Takeshi Ueki ${ }^{5,6}$, Kazuhiro Akutsu ${ }^{4}$ \& Osamu Yamamuro ${ }^{3}$

Glassy magnetic behavior has been observed in a wide range of crystalline magnetic materials called spin glass. Here, we report spin glass behavior in a structural glass of a magnetic ionic liquid, $\mathrm{C} 4 \mathrm{mimFeCl}$. Magnetization measurements demonstrate that an antiferromagnetic ordering occurs at $T_{\mathrm{N}}=2.3 \mathrm{~K}$ in the crystalline state, while a spin glass transition occurs at $T_{\mathrm{SG}}=0.4 \mathrm{~K}$ in the structural glass state. In addition, localized magnetic excitations were found in the spin glass state by inelastic neutron scattering, in contrast to spin-wave excitations in the ordered phase of the crystalline sample. The localized excitation was scaled by the Bose population factor below $T_{\mathrm{SG}}$ and gradually disappeared above $T_{\mathrm{SG}}$. This feature is highly reminiscent of boson peaks commonly observed in structural glasses. We suggest the "magnetic" boson peak to be one of the inherent dynamics of a spin glass state.
\end{abstract}

Disordered magnetic materials often display spin freezing at finite temperature and are referred to as spin glasses $(\mathrm{SGs})^{1-3}$. Theoretical attempts to model these systems have been made since the 1970s. It is generally accepted that randomness, frustration, and competing interaction are essential ingredients for SGs. These ingredients give rise to a complex energy landscape, many metastable valleys, or low-lying states in a phase space. Further, over-barrier hopping, which is time-, temperature- and magnetic-field-dependent, can occur between these valleys. In this respect, intrinsic slow relaxation takes place with a wide distribution of relaxation times. These include aging, memory, and rejuvenation effects, which have been studied extensively. However, the excitation characteristics to the metastable states are little understood. Is there an intrinsic excitation of the SG state?

Thus far, SG-like behavior has been reported in a variety of magnetic materials, including dilute magnetic alloys, mixed-phase magnetic oxides, intermetallics, and frustrated magnets, among others. Most target materials were crystalline, and SG states often appeared in proximity to long-range magnetic order phases. As the spin dynamics of crystalline materials are investigated, intrinsic spin dynamics that stemmed from the short-range magnetic order could remain even in the SG regime. To explore excitations inherent to the SG state experimentally, non-crystalline materials should be suitable.

A typical example of amorphous SGs is given in metallic glasses, for instance, multi-component alloys ${ }^{4,5}$ and binary intermetallic compounds synthesized by mechanical milling ${ }^{6}$. Some multi-component alloys are reentrant SGs in which a ferromagnetic ordered phase exists above the SG phase. In binary compounds, the possibility of microseparation is not excluded. Thus, short-range magnetic order can appear in these SG phases. In this article, we report a new type of SG material, a magnetic ionic liquid (MIL), $\mathrm{C} 4 \mathrm{mimFeCl}_{4}$ (1-butyl-3-methylimidazolium tetrachloroferrate), that is nonmetallic, contains only one kind of magnetic element, and is completely vitrified without mechanical treatment.

MILs are a class of ionic liquids that exist in liquid state around room temperature and are one of the most attractive subjects in current materials science ${ }^{7}$. The paramagnetic behavior of $\mathrm{C} \mathrm{mimFeCl}_{4}$ was first demonstrated by Hayashi et al. in $2004^{8}$. $\mathrm{C}_{\mathrm{mimFeCl}}$ comprises a large organic cation and a magnetic anion (Fig. 1a). After the discovery of the magnetic responsiveness of $\mathrm{C} 4 \mathrm{mimFeCl}_{4}, \mathrm{MILs}$ have gained considerable attention owing to their versatile applications ${ }^{9}$. MILs solely contain ions, and hence are entirely distinct from conventional magnetic fluids, which are colloidal liquids made of ferromagnetic nanoparticles suspended in nonmagnetic fluids. Most researches focus on their liquid properties and studies on low-temperature magnetic properties are

\footnotetext{
${ }^{1}$ J-PARC Center, Japan Atomic Energy Agency, Tokai, Ibaraki 319-1195, Japan. ${ }^{2}$ Division of Materials Science and Chemical Engineering, Faculty of Engineering, Yokohama National University, Yokohama, Kanagawa 240-8501, Japan. ${ }^{3}$ Institute for Solid State Physics, University of Tokyo, Kashiwa, Chiba 277-8581, Japan. ${ }^{4}$ Comprehensive Research Organization for Science and Society, Tokai, Ibaraki 319-1106, Japan. ${ }^{5}$ National Institute for Materials Science, Tsukuba, Ibaraki 305-0044, Japan. ${ }^{6}$ Graduate School of Life Science, Hokkaido University, Sapporo, Hokkaido 060-0810, Japan. ${ }^{\circledR}$ email: maiko.kofu@j-parc.jp; watanuki-ryuta-sm@ynu.ac.jp
} 


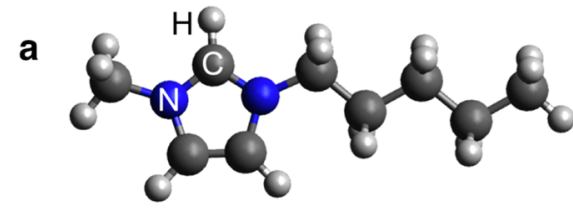

1-butyl-3-methylimidazolium (C4mim)

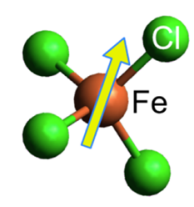

tetrachloroferrate $\left(\mathrm{FeCl}_{4}\right)$
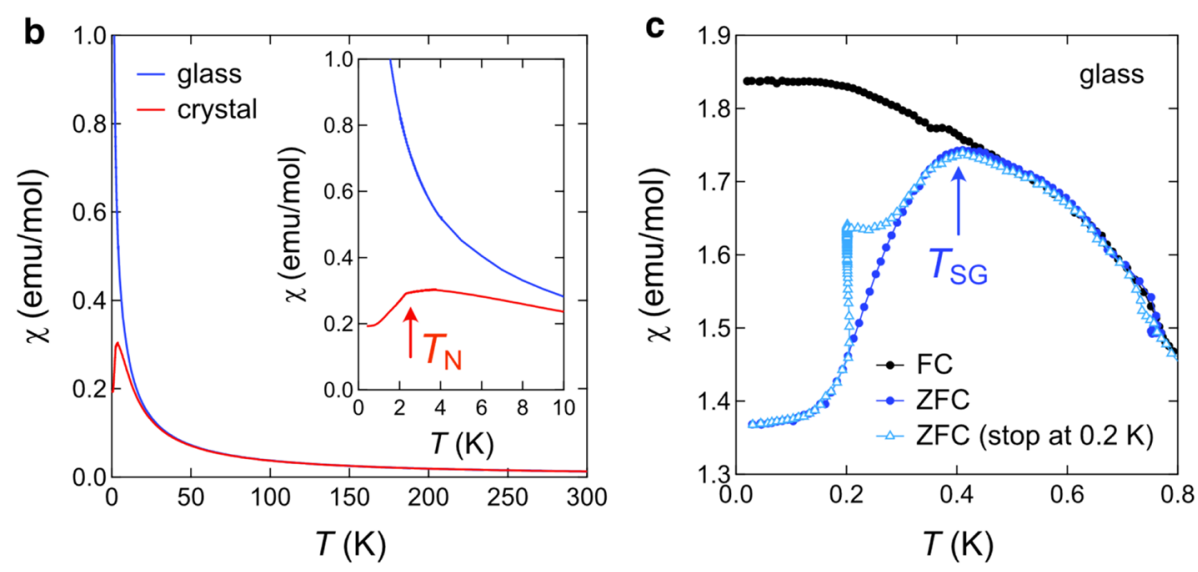

Figure 1. Magnetic properties of a magnetic ionic liquid (MIL). (a) Molecular structure of the MIL $\mathrm{C}_{4} \mathrm{mimFeCl}_{4}$, where the $\mathrm{FeCl}_{4}{ }^{-}$anion has a spin of 5/2. (b) Temperature dependence of magnetic susceptibility in glassy and crystalline $\mathrm{C}_{4} \mathrm{mimFeCl}_{4}$. Inset shows an enlarged plot below $10 \mathrm{~K}$. (c) Zero-field-cooled (ZFC) and field-cooled (FC) susceptibilities in the glassy state on heating at $T \leq 0.8 \mathrm{~K}$. Open triangles represent magnetization with an intermittent stop for $50 \mathrm{~min}$ at $T=0.2 \mathrm{~K}$. The glassy sample displays a spin glass transition at $T_{\mathrm{SG}}=0.4 \mathrm{~K}$, while the crystalline one exhibits an antiferromagnetic ordering at $T_{\mathrm{N}}=2.3 \mathrm{~K}$. All the susceptibility data were obtained under an applied magnetic field of 100 Oe.

quite limited. One remarkable feature of $\mathrm{C}_{4} \mathrm{mimFeCl}_{4}$ is that either a crystalline or glassy state can be created depending on the thermal history. The glass transition and melting temperatures of $\mathrm{C}^{2} \mathrm{mimFeCl}_{4}$ are $182 \mathrm{~K}$ and $265 \mathrm{~K}$, respectively ${ }^{10}$. We have investigated the magnetic properties of $\mathrm{C} 4 \mathrm{mimFeCl}_{4}$ in both the structural glass and crystal states by magnetization and neutron scattering measurements.

Figure $1 \mathrm{~b}$ presents magnetic susceptibilities as a function of temperature in both glassy and crystalline $\mathrm{C} 4 \mathrm{mimFeCl}_{4}$. They display paramagnetic behavior above $20 \mathrm{~K}$ and are described by the Curie-Weiss law. The Weiss temperatures and effective paramagnetic moments were estimated to be $-3.58 \mathrm{~K}$ and $5.567 \mu_{\mathrm{B}}$ for the glassy state and $-4.08 \mathrm{~K}$ and $5.564 \mu_{\mathrm{B}}$ for the crystal state (Supplementary SI-3), respectively, which are in good agreement with previous reports ${ }^{8,11}$. The obtained effective moments are slightly smaller than the theoretical value for an isotropic $\mathrm{Fe}^{3+}$ ion in the high-spin state $(S=5 / 2), 5.9 \mu_{\mathrm{B}}$. The magnetism of $\mathrm{C}_{4} \mathrm{mimFeCl}_{4}$ is dominantly governed by the antiferromagnetic (AFM) interaction between the $\mathrm{Fe}^{3+}$ spins.

In the crystal state of $\mathrm{C}_{\mathrm{mimFeCl}}$, an AFM transition occurs at $T_{\mathrm{N}}=2.3 \mathrm{~K}$ (see the inset of Fig. 1b). Meanwhile, no anomaly was observed down to $2 \mathrm{~K}$ in the structural glass state. We made further measurements down to $0.03 \mathrm{~K}$ and found that the structural glass of $\mathrm{C} 4 \mathrm{mimFeCl}_{4}$ exhibits an SG behavior (Fig. 1c). The zero-fieldcooled (ZFC) magnetization shows a maximum at $T \sim 0.4 \mathrm{~K}$ which we define as $T_{\mathrm{SG}}$, while the field-cooled (FC) magnetization decreases monotonously upon heating through $T_{\mathrm{SG}}$. Thus, there is a significant difference between the $\mathrm{ZFC}$ and $\mathrm{FC}$ magnetizations below $T_{\mathrm{SG}}$, which is usually observed around the SG transition. Furthermore, we investigated the waiting-time effect, which is a unique characteristic of the SG state ${ }^{12-15}$. After the ZFC process, the sample was heated to $0.2 \mathrm{~K}\left(<T_{\mathrm{SG}}\right)$ and remained at this temperature for $t_{\mathrm{w}}=50 \mathrm{~min}$. As $t_{\mathrm{w}}$ progressed, the magnetization increased, suggesting that the system relaxes to more stable states with lower energies. When heating is resumed, the magnetization increases again, but more gradually than the ZFC magnetization without the intermittent stops (open triangles in Fig. 1c). This behavior is referred to as the aging effect characterizing a spin freezing phenomenon. The time evolution of the magnetization in the aging regime was also investigated and is shown in Supplementary SI-4. The non-Debye relaxation process was demonstrated, suggesting a broad distribution of relaxation times.

To gain a deeper insight into the magnetic behavior within $\mathrm{C} 4 \mathrm{mimFeCl}$, we performed neutron scattering experiments, which can provide energy $(\hbar \omega)$ - and momentum $(Q)$-resolved information. Here, deuterated $\mathrm{C} 4 \mathrm{mimFeCl}_{4}$ was used to detect magnetic scattering without disturbance of strong incoherent scattering from $\mathrm{H}$ atoms (Supplementary SI-5). Figure 2 shows the $Q$-dependence of the scattering intensity at $\hbar \omega=0$, namely, the diffraction patterns. In the crystalline sample, additional magnetic Bragg peaks, indicated by the arrows in Fig. 2a, were observed at low temperatures. It is evident from Fig. $2 \mathrm{~b}$ that the magnetic peaks appear below $T_{\mathrm{N}}=2.3 \mathrm{~K}$, which is compatible with the magnetic susceptibility data. On the other hand, the diffraction patterns of the 

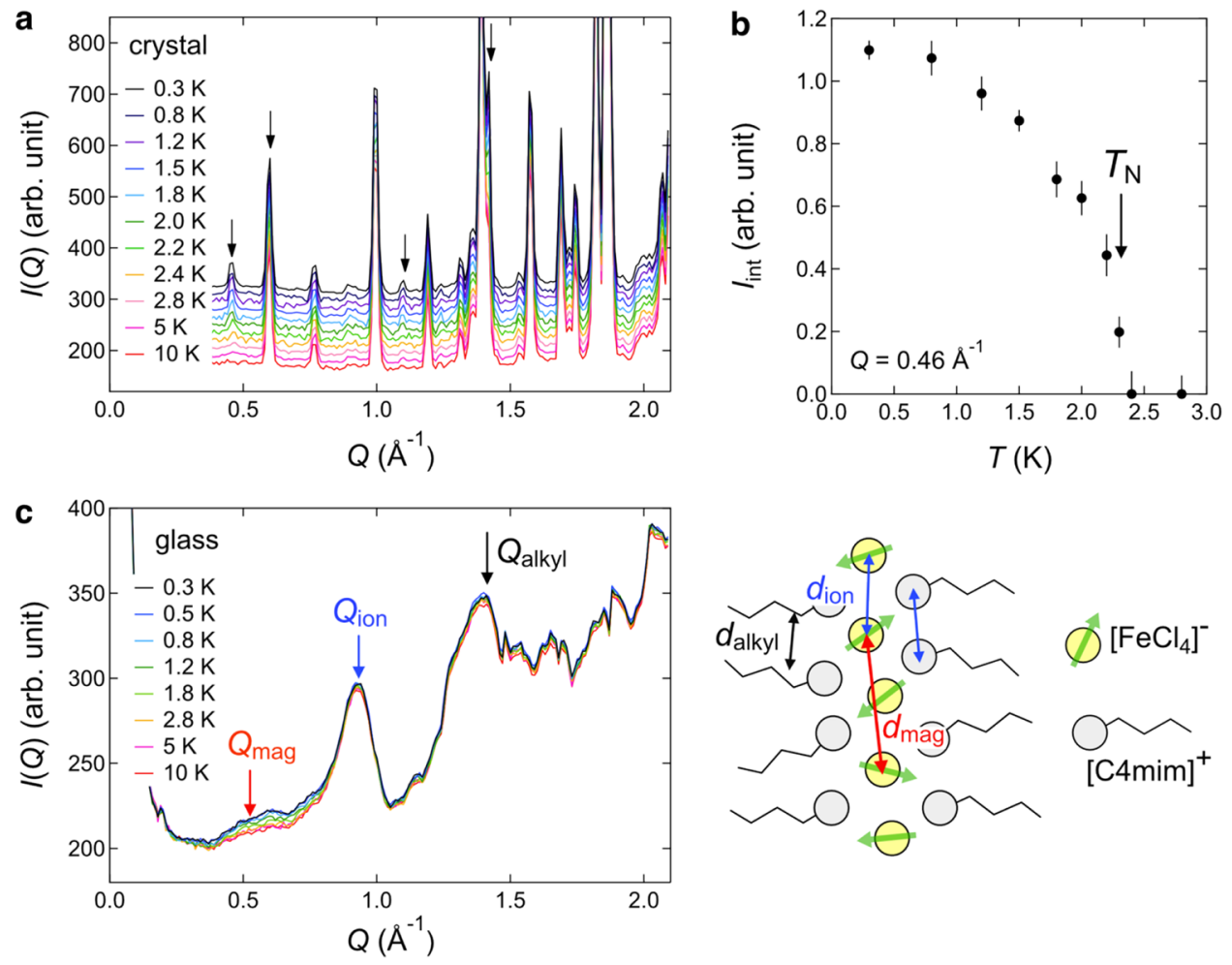

Figure 2. Diffraction patterns of $\mathrm{C}_{4} \mathrm{mimFeCl}_{4}$. (a) Diffraction patterns of crystalline $\mathrm{C}_{4} \mathrm{mimFeCl}_{4}$ between 0.3 and $10 \mathrm{~K}$. Data are shifted upward for clarity. Arrows indicate the magnetic Bragg peaks that appeared below $T_{\mathrm{N}}$. (b) Temperature dependence of integrated intensity of the magnetic Bragg peak at $Q=0.46 \AA^{-1}$. (c) Diffraction patterns of the structural glass of $\mathrm{C}_{4} \mathrm{mimFeCl}_{4}$ and schematic of the molecular and magnetic correlations with specific distances $(d=2 \pi / Q)$.

glassy sample constitute broad peaks (Fig. 2c). Those at $0.9 \AA^{-1}\left(=Q_{\text {ion }}\right)$ and $1.4 \AA^{-1}\left(=Q_{\text {alkyl }}\right)$ have commonly been observed in imidazolium-based ionic liquids ${ }^{16}$, which have local lamellar structures comprising ionic layers (anions and the charged core parts of cations) and nonionic alkyl chain layers. Moreover, the above peaks are attributed to correlations between the anions (or charged core parts) in the ionic layers and between the alkyl chains in the nonionic layers, respectively (see the illustration in Fig. 2c). In addition to these structural peaks, temperature-dependent signals were found in the $Q$ range of $0.3-0.9 \AA^{-1}$. This diffuse signal increased upon cooling, suggesting the development of magnetic correlations. The center position of the diffuse peak $\left(=Q_{\mathrm{mag}}\right)$ is approximately half of the $Q$ value of the structural peak $\left(Q_{\text {ion }}=0.93 \AA^{-1}\right)$, indicating the AFM correlation between $\mathrm{Fe}^{3+}$ spins in $\mathrm{FeCl}_{4}{ }^{-}$anions. It is also noted that the magnetic diffuse peak in the structural glass appears near the magnetic Bragg peaks in the crystalline sample, suggesting a similar local spin configuration in both the SG state and AFM phase (Supplementary SI-6).

We next describe the dynamical properties of $\mathrm{C} 4 \mathrm{mimFeCl}_{4}$. Color contour maps of the dynamical structure factor $S(Q, \omega)$ are shown in Fig. 3. A distinct difference in the excitation spectra can be recognized between the glassy and crystal states. The glassy $\mathrm{C}_{\mathrm{mimFeCl}}$ exhibits a broad low-energy magnetic excitation at $0.3 \mathrm{~K}\left(<T_{\mathrm{SG}}\right)$ (Fig. 3a). The scattering signal is enhanced at $Q \sim 0.5 \AA^{-1}$ and $\hbar \omega \sim 0.1 \mathrm{meV}$. Above $T_{\mathrm{SG}}$, the magnetic scattering is still clearly visible and its intensity is stronger near $\hbar \omega=0 \mathrm{meV}$ rather than near $0.1 \mathrm{meV}$ (Supplementary SI-7), which is suggestive of the emergence of the magnetic relaxation process (quasielastic scattering). More specifically, the system exhibits a liquid-like behavior. In contrast, spin-wave excitations with a gap attributed to magnetic anisotropy were clearly observed in the AFM order phase of the crystalline sample (Fig. 3b). The excitations extended up to $0.5 \mathrm{meV}$, suggesting that the magnitude of magnetic interaction between the $\mathrm{Fe}^{3+}$ spins is of the order of $0.1 \mathrm{meV}$. The excitation spectrum drastically changes above $T_{\mathrm{N}}$ (Fig. S8); magnetic scattering extends over a wide $\hbar \omega$ - $Q$ region. In other words, the $\mathrm{Fe}^{3+}$ spins fluctuate rapidly and the spin-spin correlations become weak, which is typical behavior for a magnetic order-disorder transition.

The spin dynamics of the SG state is summarized in Fig. 4. The raw energy spectra, results of the crystal state, and contribution of nuclear scattering are discussed in Supplementary SI-8 and SI-9. A striking result was found in the temperature evolution of the spectra corrected for the Bose thermal population factor, $\chi^{\prime \prime}(Q, \omega)=S(Q, \omega) /$ $(1+\langle n\rangle)$ with $\langle n\rangle=\left[\exp \left(\hbar \omega / k_{\mathrm{B}} T\right)-1\right]^{-1}$. The spectrum at $0.3 \mathrm{~K}$ exhibits a peak at $\sim 0.1 \mathrm{meV}$ with a high energy tail and remains almost the same at $0.5 \mathrm{~K}$ (Fig. $4 \mathrm{a}$ ), although a difference was observed in the uncorrected $S(Q, \omega)$ between $0.3 \mathrm{~K}$ and $0.5 \mathrm{~K}$ (Fig. S9). These data are taken in the measurement condition with an energy resolution of $26 \mu \mathrm{eV}$. To confirm this experimental observation, the spectra down to $0.02 \mathrm{meV}$ were investigated using another spectrometer with a fine resolution of $3.7 \mu \mathrm{eV}$. Figure $4 \mathrm{~b}$ shows the energy spectra below $T_{\mathrm{SG}}$ obtained 
glass

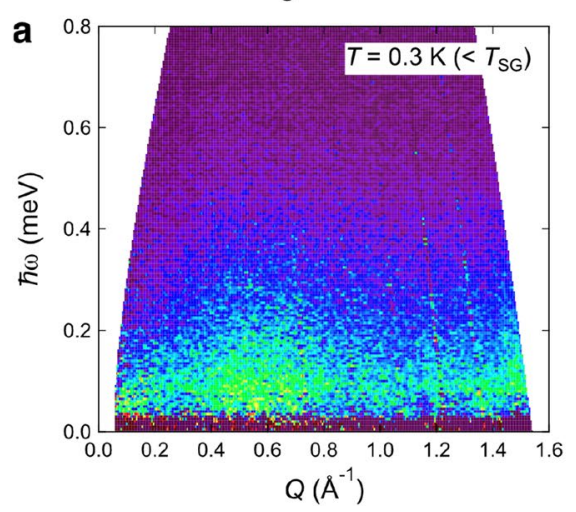

crystal

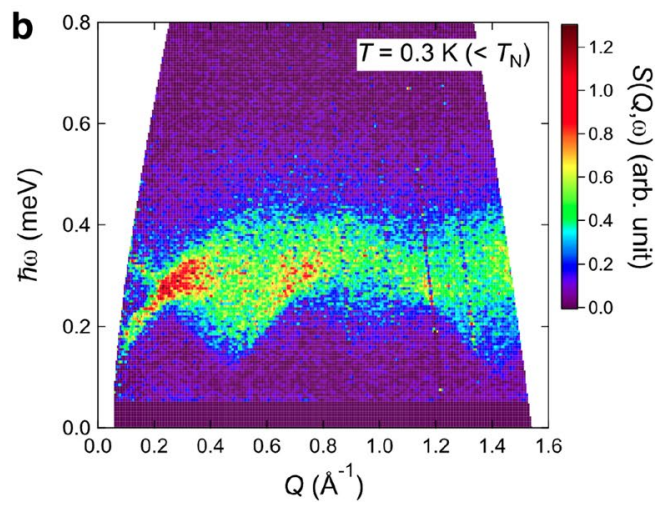

Figure 3. Magnetic excitations of $\mathrm{C}_{4} \mathrm{mimFeCl}_{4} \cdot(\mathbf{a}, \mathbf{b})$ Energy $(\hbar \omega)$-momentum $(Q)$ maps of scattering intensities of (a) glass and (b) crystal states at $T=0.3 \mathrm{~K}$.
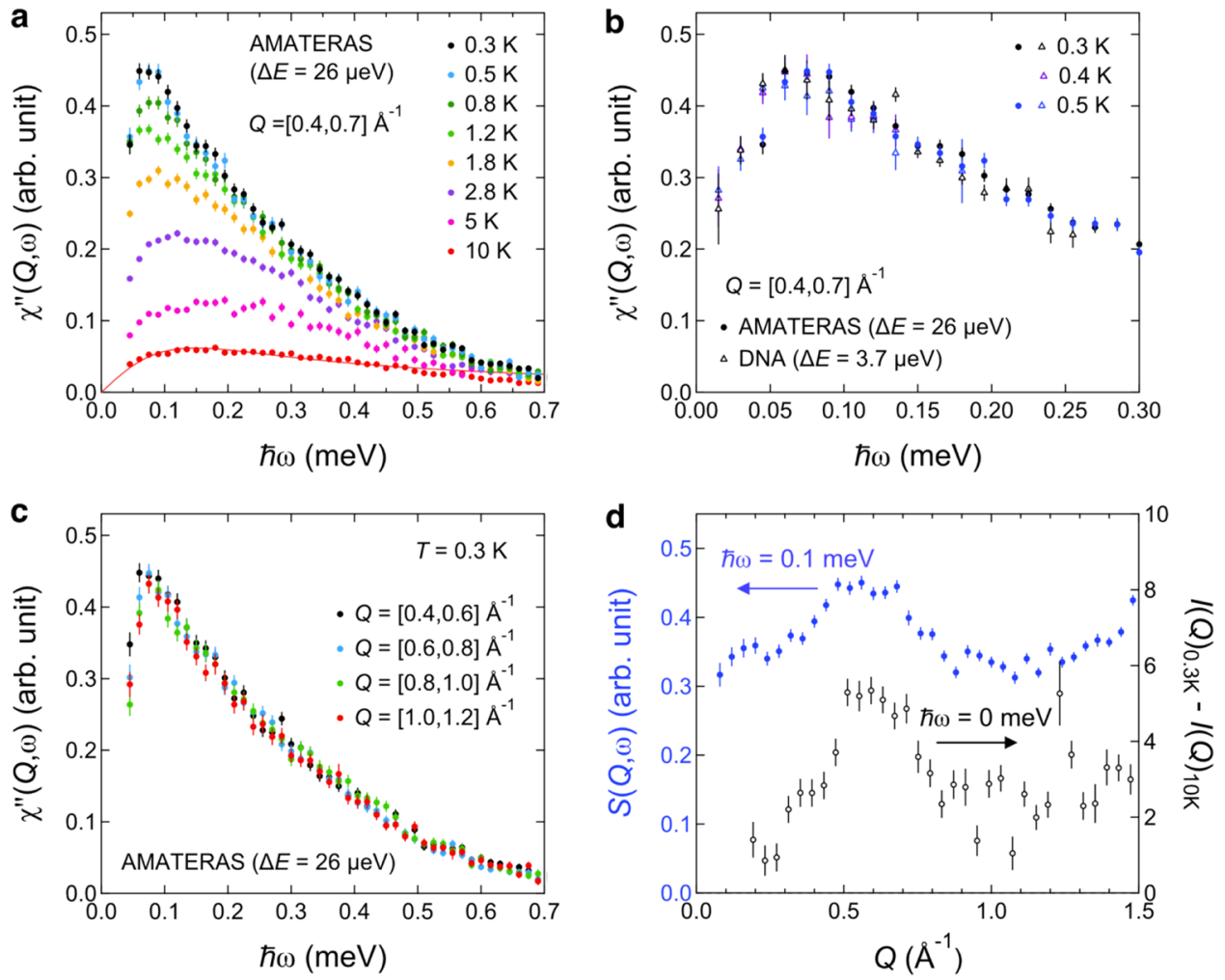

Figure 4. Energy, momentum and temperature dependence of magnetic excitation of glassy $\mathrm{C}_{4} \mathrm{mimFeCl}_{4}$. (a) Energy spectra corrected for the Bose factor, $\chi^{\prime \prime}(Q, \omega)$, in the glassy state in the temperature range of $0.3-10 \mathrm{~K}$. Red solid curve represents the result of fit with the Debye relaxation model (see main text). (b) Energy spectra below $T \approx T_{\mathrm{SG}}$ obtained using two spectrometers with different energy resolutions $(\Delta E)$. (c) Energy spectra at $T=0.3 \mathrm{~K}$ with different $Q$, where scale factors are multiplied for comparison. (d) Constant- $\hbar \omega$ cuts at $0.1 \pm 0.04 \mathrm{meV}$ (excitation peak) and $0 \pm 0.015 \mathrm{meV}$ (elastic scattering) for the $0.3 \mathrm{~K}$ data, where the magnetic elastic scattering was obtained by subtracting the data at $10 \mathrm{~K}$.

with the two spectrometers. As a result, the excitation spectrum has a maximum at $0.06 \mathrm{meV}$ and is scaled by the Bose factor below $T \approx T_{\mathrm{SG}}$.

As the temperature increases above $T_{\mathrm{SG}}$, the spectrum significantly changes (Fig. 4a). The magnitude of $\chi "(Q, \omega)$ decreases and the peak top position shifts toward the higher energy side. This result indicates that some sort of magnetic relaxation process is activated above $T_{\mathrm{SG}}$ and accelerates with increasing temperature. The spectrum at $10 \mathrm{~K}$ was fitted with a Debye relaxation model (the red solid curve in Fig. $4 \mathrm{a}), \chi^{\prime \prime}(\omega)=\chi^{\prime} \Gamma \hbar \omega /$ $\left[(\hbar \omega)^{2}+\Gamma^{2}\right]$, where $\chi^{\prime}$ is the static susceptibility and $\Gamma$ is the relaxation rate. The value of $\Gamma$ derived from the fit is 
0.149 (4) $\mathrm{meV}$, which corresponds to the relaxation time of 4.43(12) ps. The Debye relaxation does not completely reproduce the actual spectrum at $10 \mathrm{~K}$, suggesting a distribution of relaxation times. Details of the magnetic relaxation processes in $\mathrm{C} 4 \mathrm{mimFeCl}_{4}$, such as its temperature evolution and deviation from Debye behavior, will be presented in a future paper.

The energy spectrum at several $Q$ positions is shown in Fig. 4c, where suitable scale factors are multiplied to compare the spectral shapes. Apparently, the spectral shape is independent of $Q$, suggesting that the magnetic excitation is a nondispersive localized mode. Given that spin clusters with short-range AFM correlations are formed in the SG state, magnons are enclosed in the clusters, giving rise to localized magnetic excitations. The excitation intensity at $\hbar \omega=0.1 \mathrm{meV}$ is enhanced at $Q \sim 0.6 \AA^{-1}$, at which a magnetic diffuse peak appears in the elastic channel (Fig. $4 \mathrm{~d}$ ). The magnetic scattering at $\hbar \omega=0$ and $0.1 \mathrm{meV}$ stem from common spin correlations in the SG state.

We mention here that the observed feature is distinct from magnetic excitations in nanomagnets exhibiting a superparamagnetic behavior. Sharp and well-defined excitation peaks were observed in the nanomagnets, such as $\mathrm{Mn}_{12}$-acetate ${ }^{17}$, having a single type of spin cluster. The temperature dependence of the excitation intensity is described by the Boltzmann distribution, in contrast to $\mathrm{C} 4 \mathrm{mimFeCl} \mathrm{F}_{4}$ whose intensity is scaled by the Bose factor; upon heating, the scattering intensity in $\hbar \omega>0$ decreases in the nanomagnets, while increases in $\mathrm{C}_{\mathrm{mimFeCl}} \mathrm{mim}_{4}$ (see Fig. S9). The slow relaxation of nanomagnets is caused by double-well potential and hence there are two stable states. On the other hand, the dynamics of spin glass is characterized by the multi-valley energy landscape. The Bose-scaled broad excitation observed in $\mathrm{C} \mathrm{mimFeCl}_{4}$ is suggestive of the complex energy landscape with a multitude of metastable states.

The localized magnetic excitation observed below $T_{\mathrm{SG}}$ is highly reminiscent of the "boson peak" ${ }^{18}$ universally observed in structural glasses below the glass transition temperatures in neutron and Raman spectroscopies. The peak energy is located at a few meV and does not vary with $Q$. Additionally, the excitation intensity is enhanced at the structural peak positions, and is scaled by the Bose factor, indicating the excitations are of harmonic oscillators. The microscopic origin of the boson peak is, however, still under debate. The low-energy magnetic excitation in the $\mathrm{SG}$ state of $\mathrm{C}_{4} \mathrm{mimFeCl}_{4}$ is similar to the structural boson peak and could be termed the "magnetic boson peak". Incidentally, the structural boson peak is also seen in $\mathrm{C} \mathrm{mimFeCl}_{4}$. An excitation peak at $2.6 \mathrm{meV}$ was found at higher $Q$ and higher temperatures than the magnetic boson peak (Supplementary SI-10). The energy of the structural boson peak is roughly 40 times larger than that of the magnetic one because the molecular interaction is much stronger than the magnetic one in this system.

It is important to make a comparison with other SG systems studied by neutron scattering. A dilute magnetic alloy $\mathrm{Cu}_{1-x} \mathrm{Mn}_{x}$, known as a prototypical classical SG system, has been intensively investigated ${ }^{19-22}$. Magnetic scattering appears at $Q$ positions corresponding to ferromagnetic clustering and spin-density-wave above and below $T_{\mathrm{SG}}$. The scattering around the magnetic diffuse peak positions is centered at $\hbar \omega=0$ and extends to energies of $\sim 10 \mathrm{meV}$. The observed spectra were analyzed as a quasielastic scattering and explained by a model of dynamically fluctuating spin clusters with a broad distribution of relaxation times. No evidence for magnetic excitations has been adduced.

Spin dynamics of some metallic alloys exhibiting a reentrant SG behavior have also been studied ${ }^{23-26}$. Spin waves and quasielastic scattering are observed in the FM phase above $T_{\mathrm{SG}}$. Below $T_{\mathrm{SG}}$, the central peak at $\hbar \omega=0$, or a resolution-limited quasielastic peak, is dominant and spin-wave-like excitations sometimes appear due to ferromagnetically correlated magnetic clusters. Examining modern SG materials, including high-Tc cuprates ${ }^{27,28}$, heavy fermions ${ }^{29}$, and frustrated magnets ${ }^{30}$, spin excitations are often observed in their SG states. Although the excitations are modified in the SG states, they exist even outside the SG phase. Therefore, excitations inherent in SG would be invisible or absent in SG states close to magnetic order phases.

To our knowledge, a Bose-scaled localized excitation was reported only in a magnetic quasicrystal $\mathrm{Zn}-\mathrm{Mg}$ $\mathrm{Tb}^{31}$. The energy spectrum and complicated $Q$-structure are well-described by a dodecahedral spin cluster model. The result is similar to that of the spin dynamics in structurally glassy $\mathrm{C} 4 \mathrm{mimFeCl}$. It, however, should be noted that the spectrum of $\mathrm{C} 4 \mathrm{mimFeCl}_{4}$ displays a much broader tail in the high energy side (Fig. 3a). This could be attributed to a number of spin clusters with different sizes and spin configurations.

Considering theoretical calculations, many attempts have been made to derive a spin-wave spectrum, predominantly for metallic systems, in the early stage of SG research ${ }^{1,3}$. Most calculations, however, have failed to detect spin waves in the SG regime except some specific models such as a planar $S_{G}^{32,33}$. Meanwhile, a computer simulation for dilute Ruderman-Kittel-Kasuya-Yosida-coupled spin systems with Heisenberg spins (e.g., $\mathrm{Cu}_{1-x} \mathrm{Mn}_{x}$ ) has shown that the excitation spectrum extends continuously from zero to the interaction energy of nearest-neighbor pairs and exhibits a peak in a low energy range. The spectrum can reproduce the specific heat of $\mathrm{Cu}_{1-x} \mathrm{Mn}_{x}(x \sim 0.01)$ by treating the excitations as bosons ${ }^{34,35}$. In fact, the calculated spectrum resembles that observed in $\mathrm{C} 4 \mathrm{mimFeCl}_{4}$, though the model used is based on an fcc lattice and any atomistic disorder is not considered. A recent theoretical paper also suggests localized soft plastic modes whose excitation intensity reaches zero in proportion to $\omega^{4}$ in a three-dimensional Heisenberg $\mathrm{SG}^{36}$. Therefore, we consider that the localized magnetic excitation is one of the inherent dynamics in SGs and can be observed in other spin glasses separated from magnetically ordered phases. Reinvestigation of SGs such as dilute magnetic alloys is required to clarify universality of the magnetic boson peak.

Finally, we briefly discuss the magnetic interaction within $\mathrm{C} 4 \mathrm{mimFeCl}$. Magnetic structures of imidazoliumbased MILs have been investigated by neutron diffraction ${ }^{37-41}$. Typically, they have two-dimensional structure constituting anion and cation layers. Here, the main magnetic couplings are via superexchange anion-anion interactions $(\mathrm{Fe}-\mathrm{Cl} \cdots \mathrm{Cl}-\mathrm{Fe})$. As the sign of the interaction is determined by the superexchange angles, both

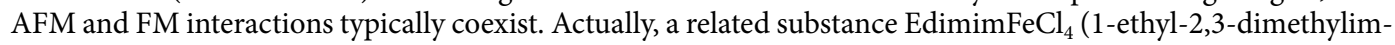
idazolium tetrachloroferrate) has two intraplane interactions, nearest-neighbor FM and next-nearest-neighbor AFM interactions, and an interplane AFM interaction ${ }^{40}$. The structural analysis for $\mathrm{C}^{4} \mathrm{mimFeCl}_{4}$ has not yet been 
succeeded because the crystalline sample obtained by annealing has strong preferred orientations. Nevertheless, one can naturally consider that both AFM and FM interactions coexist in the structural glass of C4mimFeCl with atomic disorder. In this conjecture, spin clusters can be formed via the interactions below $T_{\mathrm{SG}}$, generating a multitude of localized magnetic modes whose energy is determined by the spin configuration of each spin cluster. Consequently, this system exhibits Bose-scaled broad magnetic excitations with a high energy tail.

\section{Methods}

Sample preparation and characterization. Hydrogenated $\mathrm{C} 4 \mathrm{mimFeCl}$ ( $\mathrm{h}-\mathrm{C} 4 \mathrm{mimFeCl}$ ) with purity specified as $>98 \%$ was purchased from Tokyo Chemical Industry and used for magnetization measurements and a neutron scattering experiment to investigate vibrational excitations. The deuterated analogue (d-C4mimFeCl 4$)$ was used to probe for magnetic scattering, to avoid strong incoherent scattering contribution from $\mathrm{H}$ atoms. $\mathrm{d}-\mathrm{C} 4 \mathrm{mimFeCl}_{4}$ was prepared via the procedure as follows. 1-Butyl-3-methylimidazolium chloride $(\mathrm{C} 4 \mathrm{mimCl})$ was first synthesized using a literature method with modifications ${ }^{42}$. Reagents: 1 -methylimidazole- $d_{6}(98 \%$ atom $\mathrm{D}, \mathrm{CDN}$ isotopes), 1-chlorobutane- $d_{9}(98 \%$ atom $\mathrm{D}, \mathrm{CDN}$ isotopes), iron(III) chloride (98\%, Alfa Aesar), super dehydrated toluene (99.5\%, FUJIFILM Wako Pure Chemical Co., Ltd.), dichloromethane (99.5\%, FUJIFILM Wako Pure Chemical Co., Ltd.), and deuterium oxide (99.9\%- $d$, Sigma-Aldrich).

In an argon-filled glove bag, 1-methylimidazole- $d_{6}(2.0 \mathrm{~g}, 22.7 \mathrm{mmol})$ was dissolved in dehydrated toluene $(10 \mathrm{~mL}) .1$-Chlorobutane- $d_{9}(2.0 \mathrm{~g}, 19.7 \mathrm{mmol})$ was then added and the mixture was stirred at $20^{\circ} \mathrm{C}$ with argon bubbling for $10 \mathrm{~min}$. After, the resulting mixture was refluxed in an oil bath at $120^{\circ} \mathrm{C}$ for 7 days. When the reaction was complete, the solvent was cooled to $20^{\circ} \mathrm{C}$ and evaporated to dryness under reduced pressure. The crude product was washed a third time with dichloromethane $(30 \mathrm{~mL})$, and the remaining solution was evaporated to dryness. The yield of deuterated $\mathrm{C} 4 \mathrm{mimCl}$ was $38.2 \%$ (1.53 g). The deuteration level was estimated to be greater than $99 \%$ by nuclear magnetic resonance and electrospray ionization mass spectrometry (Supplementary SI-1).

C4mimCl- $d_{15}(0.97 \mathrm{~g}, 5.1 \mathrm{mmol})$ and $\mathrm{FeCl}_{3}(0.865 \mathrm{~g}, 5.3 \mathrm{mmol})$ were dissolved in $10 \mathrm{~mL}$ of $\mathrm{D}_{2} \mathrm{O}$, and the mixture was stirred at $20^{\circ} \mathrm{C}$ for $10 \mathrm{~min}$. Then, the mixture was filtrated through a polypropylene filter $(0.45 \mu \mathrm{m}$ pore size) to remove the small amount of insoluble material. The filtered solution was evacuated at $70{ }^{\circ} \mathrm{C}$ for $24 \mathrm{~h}$ to dryness under reduced pressure. The yield of $\mathrm{d}-\mathrm{C} 4 \mathrm{mimFeCl}_{4}$ was $94.3 \%(1.73 \mathrm{~g})$.

The thermodynamic and paramagnetic properties of both $\mathrm{h}$ - and $\mathrm{d}-\mathrm{C} 4 \mathrm{mimFeCl} \mathrm{F}_{4}$ were examined by differential scanning calorimetry (DSC) and magnetic susceptibility measurements. Deuterium isotope effects on these properties were not confirmed (Supplementary SI-2).

Magnetization measurements. The magnetizations of $\mathrm{C} \mathrm{mimFeCl}_{4}$ were measured using a SQUID magnetometer (MPMS-XL, Quantum Design Co.) in the temperature range of 1.8-300 K. For the measurement sample with SQUID, liquid $\mathrm{C} 4 \mathrm{mimFeCl}_{4}(0.1010 \mathrm{~g})$ was put in a clear gelatin capsule. The capsule was fixed to a straw and set in the SQUID magnetometer. The dc magnetization measurements in the temperature ranges of $0.03-0.8 \mathrm{~K}$ and $0.4-3.0 \mathrm{~K}$ were performed using a capacitive Faraday magnetometer installed in a ${ }^{3} \mathrm{He}$ and a dilution refrigerator, respectively ${ }^{43}$. In the Faraday method, a small cup made of pure copper whose surface is coated with gold was prepared. The liquid $\mathrm{C} 4 \mathrm{mimFeCl}_{4}(0.0706 \mathrm{~g})$ was poured into this cup and set using Apiezon $\mathrm{N}$ grease in the measurement stage. All the magnetization data were obtained under an applied magnetic field of 100 Oe.

Neutron scattering measurements. Neutron scattering measurements were performed using a diskchopper spectrometer AMATERAS ${ }^{44}$ and a near-backscattering TOF spectrometer DNA ${ }^{45}$ at the Materials and Life Science Experimental Facility (MLF), J-PARC. The d-C4mimFeCl ${ }_{4}$ sample was used to explore magnetic scattering and the h-C $4 \mathrm{mimFeCl}_{4}$ sample for vibrational excitations. The deuterated sample was loaded into an annular Al can with a diameter of $7 \mathrm{~mm}$, while the hydrogenated sample was placed into a concentric doublecylinder $\mathrm{Al}$ can with a diameter of $14 \mathrm{~mm}$ and a thickness of $0.5 \mathrm{~mm}$. To control the temperature of the samples, a ${ }^{3} \mathrm{He}$ refrigerator, top-loading closed-cycle ${ }^{4} \mathrm{He}$ refrigerator, and dilution refrigerator were used. The glassy $\mathrm{C} 4 \mathrm{mimFeCl}_{4}$ was created by cooling the liquid with a rate of $1-3 \mathrm{~K} / \mathrm{min}$. The crystalline sample was obtained by the following annealing procedure. The glassy sample was first kept at $130 \mathrm{~K}$ for $2 \mathrm{~h}$ and then heated to $230 \mathrm{~K}$, which is $35 \mathrm{~K}$ below the melting temperature. The crystallization occurred at $230 \mathrm{~K}$ within $1 \mathrm{~h}$, which was confirmed by the growth of the Bragg diffraction peaks.

In the measurements on AMATERAS for magnetic scattering, the incident neutron energies used were $E_{\mathrm{i}}=3.13$ and $1.68 \mathrm{meV}$ with corresponding energy resolutions $(\Delta E)$ of 62 and $26 \mu \mathrm{eV}$ (full width at half maximum of the elastic peaks), respectively. The scattering signals were recorded in the temperature range of $0.3-100 \mathrm{~K}$. The vibrational excitations were measured with $E_{\mathrm{i}}=7.74 \mathrm{meV}$ and $\Delta E=0.22 \mathrm{meV}$ at $T=7 \mathrm{~K}$ and $50 \mathrm{~K}$. The DNA spectrometer having a higher energy resolution $(\Delta E=3.7 \mu \mathrm{eV})$ was used to investigate the magnetic excitation of glassy $\mathrm{d}-\mathrm{C} 4 \mathrm{mimFeCl}_{4}$ below $\hbar \omega=0.27 \mathrm{meV}$ in the temperature range of $0.3-0.5 \mathrm{~K}$. The final neutron energy was fixed to $2.08 \mathrm{meV}$. Moreover, wavelength frame multiplication was utilized to extend the energy window while maintaining the energy resolution. All the data collected were analyzed using the software suite UTSUSEMI ${ }^{46}$. The dynamical structure factor $S(Q, \omega)$ shown in this article are obtained by subtracting elastic signals. The elastic scattering contribution is estimated by the convolution of resolution function and $I(Q)$ of the sample. The resolution functions are obtained by the data of the crystal state at $0.3 \mathrm{~K}$ for AMATERAS and vanadium standard for DNA.

Received: 11 April 2021; Accepted: 28 May 2021

Published online: 08 June 2021 


\section{References}

1. Fischer, K. H. \& Hertz, J. A. Spin Glasses (Cambridge University Press, 1991).

2. Mydosh, J. A. Spin glasses: Redux: An updated experimental/materials survey. Rep. Prog. Phys. 78, 052501 (2005).

3. Binder, K. \& Young, A. P. Spin glasses: Experimental facts, theoretical concepts, and open questions. Rev. Mod. Phys. 58, 801-976 (1986).

4. Svedlindh, P. et al. Relaxation in spin glasses at weak magnetic fields. Phys. Rev. B 35, 268-273 (1987).

5. Luo, Q., Zhao, D. Q., Pan, M. X. \& Wang, W. H. Magnetocaloric effect of Ho-, Dy-, and Er-based bulk metallic glasses in helium and hydrogen liquefaction temperature range. Appl. Phys. Lett. 90, 211903 (2007).

6. Zhou, G. F. \& Bakker, H. Spin-glass behavior of amorphous $\mathrm{Co}_{2} \mathrm{Ge}$ synthesized by mechanical milling. Phys. Rev. Lett. 72, 22902293 (1993)

7. MacFarlane, D. R., Kar, M. \& Pringle, J. M. Fundamentals of Ionic Liquids: From Chemistry to Applications (Wiley, 2017).

8. Hayashi, S. \& Hamaguchi, H. Discovery of a magnetic ionic liquid [bmim] $\mathrm{FeCl}_{4}$. Chem. Lett. 33, 1590-1591 (2004).

9. Santos, E., Albob, J. \& Irabien, A. Magnetic ionic liquids: Synthesis, properties and applications. RSC Adv. 4, 40008-40018 (2014).

10. Yamamuro, O., Inamura, Y., Hayashi, S. \& Hamaguchi, H. Glass transitions and low-frequency dynamics of room-temperature ionic liquids. AIP Conf. Proc. 832, 73-80 (2006).

11. Cruz, M. M. et al. Thermophysical and magnetic studies of two paramagnetic liquid salts: $\left[\mathrm{C}_{4} \mathrm{mim}\right]\left[\mathrm{FeCl}_{4}\right]$ and $\left[\mathrm{P}_{66614}\right]\left[\mathrm{FeCl}_{4}\right]$. Fluid Phase Equilib. 350, 43-50 (2013).

12. Mathieu, R., Jönsson, P., Nam, D. N. H. \& Nordblad, P. Memory and superposition in a spin glass. Phys. Rev. B 63, 092401 (2001).

13. Bernardi, L. W. et al. Aging of the zero-field-cooled magnetization in Ising spin glasses: Experiment and numerical simulation. Phys. Rev. Lett. 86, 720-723 (2001).

14. Vincent, E. Ageing, rejuvenation and memory: The example of spin-glasses. In Ageing and the Glass Transition. Lecture Notes in Physics Vol. 716 (eds Henkel, M. et al.) 7-60 (Springer, Berlin, 2007).

15. Samarakoon, A. et al. Aging, memory, and nonhierarchical energy landscape of spin jam. Proc. Natl. Acad. Sci. 113, 11806-11810 (2016).

16. Zhang, W. et al. Effect of cation symmetry on the morphology and physicochemical properties of imidazolium ionic liquids. J. Phys. Chem. B 115, 6572-6584 (2011).

17. Mirebeau, I. et al. Low-energy magnetic excitations of the $\mathrm{mn}_{12}$-acetate spin cluster observed by neutron scattering. Phys. Rev. Lett. 83, 628-631 (1999).

18. Nakayama, T. Boson peak and terahertz frequency dynamics of vitreous silica. Rep. Prog. Phys. 65, 1195-1242 (2002).

19. Borovik-Romanov, A. S. \& Shinha, S. K. Spin Waves and Magnetic Excitations (North-Holland, 1988).

20. Murani, A. P. Spectral distribution of relaxation times in spin glasses. Magn. Magn. Mater. 22, 271-281 (1981).

21. Tsunoda, Y., Kunitomi, N. \& Cable, J. W. Magnetic excitation in CuMn spin glass alloy. J. Appl. Phys. 57, 3753-3755 (1985).

22. Werner, S. A., Rhyne, J. J. \& Gotaas, J. A. Spin density wave magnetism in copper-manganese alloys. Solid State Commun. 57, 457-460 (1985).

23. Hennion, B., Hennion, M., Hippert, F. \& Murani, A. P. Evidence of ferromagnetic spin waves in the spin-glass state of a re-entrant Ni-Mn alloy. J. Phys. F Met. Phys. 14, 489-504 (1984).

24. Motoya, K., Shapiro, S. M. \& Muraoka, Y. Neutron scattering studies of the anomalous magnetic alloy Fe ${ }_{0.7} \mathrm{Al}_{0.3}$. Phys. Rev. B 28, 6183-6191 (1983).

25. Lequien, S., Hennion, B. \& Shapiro, S. M. Reinvestigation of the reentrant spin-glass phase of $\mathrm{Fe}_{26} \mathrm{Cr}_{74}$ by high-resolution inelastic neutron scattering. Phys. Rev. B 38, 2669-2674 (1988).

26. Aeppli, G., Shapiro, S. M., Birgeneau, R. J. \& Chen, H. S. Spin correlations and reentrant spin-glass behavior in amorphous Fe-Mn alloys. II. Dynamics. Phys. Rev. B 29, 2589-2605 (1984).

27. Matsuda, M. et al. Magnetic dispersion of the diagonal incommensurate phase in lightly doped $\mathrm{La}_{2-x} \mathrm{Sr}_{x} \mathrm{CuO}_{4}$. Phys. Rev. Lett. 101, 197001 (2008).

28. Enoki, M. et al. Spin-stripe density varies linearly with the hole content in single-layer $\mathrm{Bi}_{2+x} \mathrm{Sr}_{2-x} \mathrm{CuO}_{6+y}$ cuprate superconductors. Phys. Rev. Lett. 110, 017004 (2013).

29. Goremychkin, E. A. et al. Spin-glass order induced by dynamic frustration. Nat. Phys. 4, 766-770 (2008)

30. Ehlers, G. et al. High-resolution neutron scattering study of $\mathrm{Tb}_{2} \mathrm{Mo}_{2} \mathrm{O}_{7}$ : A geometrically frustrated spin glass. Phys. Rev. B 81, 224405 (2010).

31. Sato, T. J., Takakura, H., Tsai, A. P. \& Shibata, K. Magnetic excitations in the Zn-Mg-Tb icosahedral quasicrystal: An inelastic neutron scattering study. Phys. Rev. B 73, 054417 (2006).

32. Edwards, S. F. \& Anderson, P. W. Theory of spin glasses. II. J. Phys. F 6, 1927 (1976).

33. Huber, D. L. \& Ching, W. Y. Collective excitations in a planar model of a classical spin glass. J. Phys. C 13, 5579 (1980).

34. Walker, L. R. \& Walstedt, R. E. Computer model of metallic spin-glasses. Phys. Rev. Lett. 28, 514-518 (1977).

35. Walker, L. R. \& Walstedt, R. E. Computer model of metallic spin-glasses. Phys. Rev. B. 22, 3816-3842 (1980).

36. Baity-Jesi, M., Martín-Mayor, V., Parisi, G. \& Perez-Gaviro, S. Soft modes, localization, and two-level systems in spin glasses. Phys. Rev. Lett. 115, 267205 (2015).

37. García-Saiz, A. et al. Anion- $\pi$ and halide-halide nonbonding interactions in a new ionic liquid based on imidazolium cation with three-dimensional magnetic ordering in the solid state. Inorg. Chem. 53, 8384-8396 (2014).

38. García-Saiz, A. et al. Neutron powder diffraction study of the magnetic ionic liquid $\mathrm{Emim}\left[\mathrm{FeCl}_{4}\right]$ and its deuterated phase. J. Phys. Conf. Ser. 663, 012008 (2015).

39. de Pedro, I. et al. Dynamically slow solid-to-solid phase transition induced by thermal treatment of DimimFeCl4 magnetic ionic liquid. Phys. Chem. Chem. Phys. 18, 21881-21892 (2016).

40. González-Izquierdo, P. et al. Magnetic structure, single-crystal to single-crystal transition, and thermal expansion study of the (Edimim) $\left[\mathrm{FeCl}_{4}\right]$ halometalate compound. Inorg. Chem. 57, 1787-1795 (2018).

41. González-Izquierdo, P. et al. Crystal structure, magneto-structural correlation, thermal and electrical studies of an imidazolium halometallate molten salt: (trimim) $\left[\mathrm{FeCl}_{4}\right]$. RSC Adv. 10, 11200 (2020).

42. Akutsu, K. et al. Penetration behavior of an ionic liquid in thin-layer silica coating: Ionic liquid deuteration and neutron reflectivity analysis. Phys. B 551, 262-265 (2018).

43. Sakakibara, T., Mitamura, H., Tayama, T. \& Amitsuka, H. Faraday force magnetometer for high-sensitivity magnetization measurements at very low temperatures and high fields. Jpn. J. Appl. Phys. 33, 5067 (1994).

44. Nakajima, K. et al. AMATERAS: A cold-neutron disk chopper spectrometer. J. Phys. Soc. Jpn. 80, SB028 (2011).

45. Shibata, K. et al. The performance of TOF near backscattering spectrometer DNA in MLF. J-PARC. JPS Conf. Proc. 8, 036022 (2015).

46. Inamura, Y., Nakatani, T., Suzuki, J. \& Otomo, T. Development status of software "Utsusemi” for chopper spectrometers at MLF. J-PARC. J. Phys. Soc. Jpn. 82, SA031 (2013).

\section{Acknowledgements}

The neutron scattering experiments at the Materials and Life Science Experimental Facility (MLF), J-PARC, were performed under the approved proposals (Nos. 2016I0014, 2018B0263, 2019B0025, and 2020I0014). The 
synthesis of deuterated $\mathrm{C} 4 \mathrm{mimFeCl}_{4}$ and its characterization by DSC and MPMS were conducted at the CROSS User Experiment Preparation Labs. The preparation of the deuterated material was partly supported by the deuteration laboratory in MLF. We thank S. Hayashi for fruitful discussions and D. Wakai, M. Ishikado, R. Takahashi, and T. Yamada for their help in neutron scattering experiments and sample characterization. This work was supported by JSPS KAKENHI Grant Number JP 17K05619.

\section{Author contributions}

M.K., R.W., T.S. and O.Y. conceived the project. R.W., T.S. and O.Y performed the magnetization measurements. M. K. S. O-K. K. N. and M. M. conducted the neutron scattering experiments. T.U. and K.A. synthesized the deuterated sample. M.K. and R.W. analyzed the data. All authors contributed to writing the manuscript.

\section{Competing interests}

The authors declare no competing interests.

\section{Additional information}

Supplementary Information The online version contains supplementary material available at https://doi.org/ 10.1038/s41598-021-91619-z.

Correspondence and requests for materials should be addressed to M.K. or R.W.

Reprints and permissions information is available at www.nature.com/reprints.

Publisher's note Springer Nature remains neutral with regard to jurisdictional claims in published maps and institutional affiliations.

(c) (i) Open Access This article is licensed under a Creative Commons Attribution 4.0 International License, which permits use, sharing, adaptation, distribution and reproduction in any medium or format, as long as you give appropriate credit to the original author(s) and the source, provide a link to the Creative Commons licence, and indicate if changes were made. The images or other third party material in this article are included in the article's Creative Commons licence, unless indicated otherwise in a credit line to the material. If material is not included in the article's Creative Commons licence and your intended use is not permitted by statutory regulation or exceeds the permitted use, you will need to obtain permission directly from the copyright holder. To view a copy of this licence, visit http://creativecommons.org/licenses/by/4.0/.

(C) The Author(s) 2021 